\title{
DESENVOLVIMENTO DE UM MÓDULO DE PROGRAMAÇÃO LINEAR NO PROGRAMA OPTIMI
}

\author{
BIONDI C. O. ${ }^{1}$, VIANNA S. S. V. ${ }^{2}$, RODRIGUES M. T. M. ${ }^{3}$
}

${ }^{1}$ Universidade Estadual de Campinas, Departamento de Engenharia de Sistemas Químicos ${ }^{2}$ Universidade Estadual de Campinas, Departamento de Engenharia de Sistemas Químicos ${ }^{3}$ Universidade Estadual de Campinas, Departamento de Engenharia de Sistemas Químicos E-mail para contato: caiobiondi@gmail.com

RESUMO - Esse trabalho teve como objetivo desenvolver um novo módulo de otimização para o programa OPTIMI, software patenteado pela FEQ/UNICAMP, que até então só resolvia problemas de otimização binária. Foi desenvolvido um algoritmo que resolve problemas de otimização linear, utilizando a linguagem FORTRAN 77, baseado no método Simplex Revisado. Além disso, foram acrescentados algoritmos específicos para tratamento das matrizes de entrada como, por exemplo, normalização e esparsidade. O módulo desenvolvido foi validado através de comparações feitas com softwares comerciais disponíveis. Por fim, foi feito um estudo de caso a fim de resolver um problema real de uma refinaria de petróleo. Os resultados obtidos foram satisfatórios, já que o programa foi capaz de resolver com rapidez e precisão todos os problemas avaliados, apresentando resultados semelhantes a softwares comerciais.

\section{INTRODUÇÃO}

A indústria química passou por grandes mudanças nos últimos 30 anos. A instabilidade nos preços de energia e de matéria prima, induzida principalmente pela crise do petróleo na década de 70 , mostrou que era preciso produzir com mais tecnologia e com menos dependência energética. Maiores preocupações em relação ao impacto da produção industrial na segurança dos trabalhadores e no meio ambiente também vieram à tona a partir dessa época. Além disso, a competição por preço e qualidade dos produtos ficava cada vez mais acirrada. (Perkins, 2003)

Uma das ferramentas mais importantes da engenharia utilizada para solucionar essas novas questões é a otimização. Segundo Edgar et al. (2001), a otimização é o uso de métodos específicos para determinar o melhor custo benefício e uma solução eficiente para um problema ou projeto de um processo. Essa técnica é a maior ferramenta quantitativa usada na indústria para ajudar nas tomadas de decisão. Uma extensa variedade de problemas de projeto, construção, operação e análise de plantas químicas, bem como muitos outros processos industriais, pode ser resolvido por otimização.

A otimização permeia os campos da ciência, engenharia e administração. Na física, muitos princípios ótimos que descrevem fenômenos naturais nos campos da ótica e mecânica clássica foram enunciados. A estatística possui princípios chamados de máxima probabilidade, perda mínima e 
mínimos quadrados. Já a administração frequentemente utiliza os conceitos de máximo lucro, custo mínimo, uso máximo dos recursos e esforço mínimo.

Problemas típicos da engenharia química, no projeto ou na operação de uma planta, têm muitas soluções. A otimização consiste em escolher a melhor solução dentro do conjunto possível através de métodos quantitativos eficientes. Softwares específicos tornam essa tarefa possível e viável economicamente. Porém, para obter informações úteis dos computadores é necessária uma análise profunda do processo em questão, compreensão dos objetivos desejados e, normalmente, experiência previa.

Os benefícios adquiridos pelo uso dessas técnicas são muitos: aumento de desempenho, aumento da quantidade de produtos de interesse, redução da quantidade de contaminantes, redução do consumo de energia, redução do tempo de parada, redução do custo de manutenção, redução do desgaste de equipamentos e melhor utilização de pessoal. Além disso, apenas a identificação do objetivo, restrições e graus de liberdade do sistema trazem benefícios não mensuráveis como, por exemplo, aumento da qualidade de projeto, maior confiança na solução de problemas e tomadas de decisão mais rápidas.

\subsection{O Programa OPTIMI}

Desenvolvido durante um projeto de mestrado e posteriormente patenteado pela FEQ/UNICAMP, o programa OPTIMI possui um algoritmo de otimização binária baseado no método de Balas (Vianna, 2004). O software foi customizado para calcular o menor número de detectores de gás fazendo uma interface com softwares de fluido dinâmica computacional (CFD).

Para o aperfeiçoamento do programa, é necessário, no entanto, expandir o seu range de atuação para além de problemas discretos. Diversos casos no dia a dia de um engenheiro são contínuos e, por isso, a necessidade de criar novos módulos de otimização como, por exemplo, otimização linear e não-linear.

\section{OBJETIVOS}

Esse trabalho tem como objetivo geral o desenvolvimento de um módulo de otimização linear a ser incorporado no programa OPTIMI. Para atingir essa meta, foi necessário definir os seguintes objetivos específicos:

- Programação do algoritmo Simplex em linguagem Fortran 77;

- Validação do algoritmo através de problemas com resultados conhecidos na literatura;

- Pesquisa aprofundada de convergência em métodos computacionais;

- Estudo de caso em uma indústria operante. 


\section{9 a 22 de outubro de 2014 \\ Florianópolis/SC}

\section{DESCRIÇÃO DO TRABALHO REALIZADO}

Desenvolvimento do algoritmo: admitindo que o problema tenha solução ótima, pode ser demonstrado que essa solução se encontra entre uma das soluções básicas viáveis. O SIMPLEX é nada mais que um algoritmo capaz de, a partir de uma solução básica viável inicial, gerar novas soluções básicas viáveis cada vez melhores, até chegar a uma que não pode mais ser melhorada. (Bregalda et al. 1983).

Após a criação do algoritmo SIMPLEX por George Dantzig, diversas outras versões foram feitas a partir do mesmo princípio. De acordo com Syslo et al. (1983), a ideia é adaptar a metodologia para ser mais fácil de implementá-la em uma determinada linguagem de programação ou para resolver mais eficientemente um problema específico. Uma dessas modificações deu origem ao Simplex Revisado, que utiliza multiplicações envolvendo a inversa da matriz base para resolver o problema.

A linguagem de programação utilizada na elaboração do algoritmo SIMPLEX foi Fortran 77. Essa escolha foi feita com o intuito de manter a consistência com o módulo de otimização binária que foi desenvolvido nessa mesma linguagem, a fim de não gerar incompatibilidade entre os dois módulos.

Além disso, estão sendo implementados tratamentos na matriz inicial do problema para evitar dificuldades de convergência da solução ótima. Uma metodologia empregada permite a normalização da matriz dos coeficientes, isto é, transforma os dados em uma escala comum para que possa haver comparação de forma significativa. Outro método considerado trata os casos onde haja matrizes esparsas, aquelas em que há grande quantidade de valores zero na matriz dos coeficientes.

Validação dos resultados: durante os testes do algoritmo foram utilizados problemas de programação linear conhecidos da literatura. Bregalda (1983), Boldrini (1980), Edgar (2001) e Syslo (1983) são exemplos de autores utilizados para validação dos resultados. Nesses trabalhos são encontrados problemas de ordem de grandeza variando de matrizes $2 \times 2$ a matrizes $8 \times 5$, que permitiram evidenciar a eficácia do algoritmo, já que em todos os casos ele encontrou a mesma solução ótima obtida pelos autores estudados.

Esses mesmos casos foram utilizados como teste em um software conhecido e aceito comercialmente chamado GAMS. Esse processo foi importante para adquirir o conhecimento necessário do GAMS para comparar os resultados obtidos pelo algoritmo desenvolvido no estudo de caso realizado nesse trabalho.

Estudo de caso: foi simulado um problema real de uma indústria química a fim de testar a eficácia do software desenvolvido. O processo escolhido para realizar esse estudo foi uma refinaria de petróleo e a sua escolha foi baseada na possibilidade de criar um problema complexo com muitas variáveis e restrições. Nessa etapa do trabalho foram feitas reuniões na refinaria de Paulínia REPLAN da Petrobras para que o processo, mesmo que simplificado, fosse o mais real possível. 
A simulação foi feita para uma refinaria fictícia com capacidade de refino de até 100.000 barris de petróleo por dia. Nesse problema, há possibilidade de compra de 11 tipos diferentes de petróleos, cada um com uma composição diferente, porém conhecida com base em informações fornecidas pelas próprias empresas produtoras e disponíveis em domínio público através dos seus websites. Restrições de disponibilidade de compra desses óleos e o seu custo foram considerados nessa análise. O esquema do problema formulado pode ser observado no fluxograma da Figura 1 abaixo.

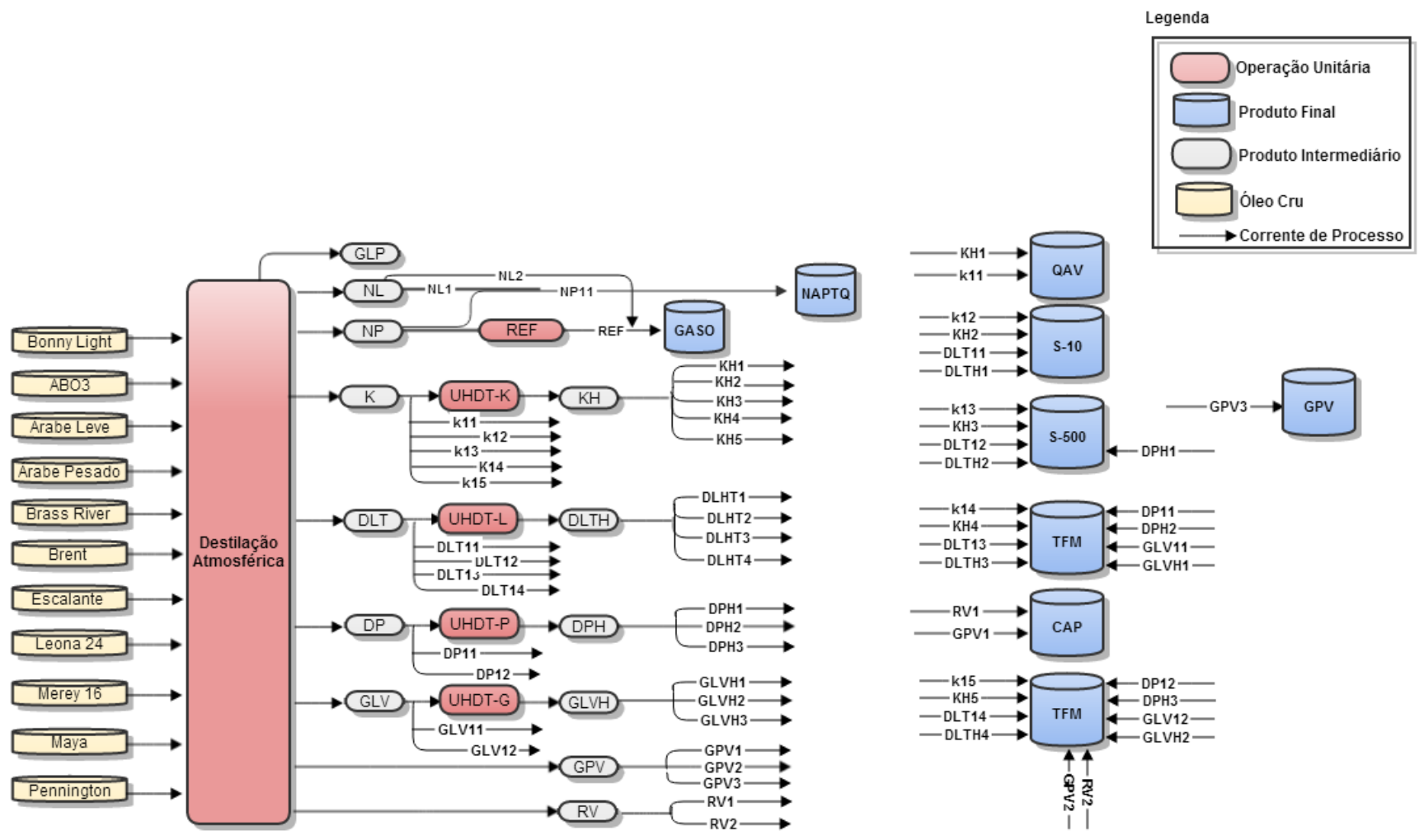

Figura 1: Esquema do problema analisado no estudo de caso.

Os resultados obtidos pelo algoritmo desenvolvido foram considerados satisfatórios, já que eles foram comparados com o software comercial GAMS. O tempo de processamento dos dados também foi considerado bom para problemas dessa ordem de grandeza, pois os resultados obtidos foram calculados na ordem de segundos.

\section{CONCLUSÕES}

Foi possível concluir o trabalho de forma satisfatória, já que o algoritmo desenvolvido foi capaz de resolver todos os problemas de programação linear encontrados na literatura e também chegou à mesma solução do software comercial GAMS no estudo de caso proposto nesse trabalho.

Para trabalhos futuros, é proposta a criação de novos módulos de otimização como, por exemplo, para os casos de problemas não lineares. Percebe-se, na engenheira química, uma grande 
quantidade de problemas não lineares e, por isso, a necessidade de novas metodologias que levem em consideração essas particularidades.

\section{REFERÊNCIAS}

BREGALDA, P.F.; OLIVEIRA A.A.F.; BORNSTEIN C.T. Introdução à programação linear. Rio de Janeiro: Editora Campus Ltda, 1983.

BOLDRINI J.L.; COSTA S.I.R.; FIGUEIREDO V.L. Álgebra Linear. São Paulo, 1980.

EDGAR T.F.; HIMMELBLAU D.M. Optimization of Chemical Processes, New York: Editora McGraw-Hill, 2001.

PERKINS J.D. Chemical Engineering - the First 100 Years. Chemical Engineering: Visions of the World, 2003.

SYSLO M.M.; DEO N.; KOWALIK J.S. Discrete Optimization Algorithms. New Jersey: Editora Prentice-Hall, 1983.

VIANNA S.S. Otimização de detectores de gás usando programação matemática e fluidodinâmica computacional. Dissertação de Mestrado, Rio de Janeiro, 2004. 